\title{
Correlación del strain longitudinal global con el grado de disfunción diastólica, factores de riesgo cardiovascular y variables del ecocardiograma 2D
}

\author{
Global longitudinal strain relation with diastolic dysfunction degree, \\ cardiovascular risk factors and 2D echocardiogram variables
}

\author{
Francisco Sánchez Lezama, ${ }^{*}$ Luis Gerardo Domínguez Carrillo, ${ }^{\ddagger}$ \\ Stephany Carolina Rivas León, § Diana Flores Peñađ
}

Citar como: Sánchez LF, Domínguez CLG, Rivas LSC, Flores PD. Correlación del strain longitudinal global con el grado de disfunción diastólica, factores de riesgo cardiovascular y variables del ecocardiograma 2D. Acta Med GA. 2021; 19 (4): 485-490. https://dx.doi.org/10.35366/102532

\section{Resumen}

Introducción: El pronóstico en pacientes con enfermedad estructural cardiovascular depende en gran manera de la calidad de la función ventricular izquierda, la medición de la deformación miocárdica (strain) es un indicador mucho más temprano y sensible de falla cardiaca. Objetivo: Correlacionar factores de riesgo cardiovascular y mediciones ecocardiográficas con el índice de deformidad longitudinal global (SLG). Material y métodos: Se estudiaron 53 pacientes con edad promedio de 59.8 años y recorrido de 22-91 años, en los que se efectuaron mediciones electrocardiográficas habituales y de SLG. Análisis estadístico: Se utilizó $\chi^{2}$, t de Student y razón de desventaja (odds ratio) para valorar la relación de las variables con SLG. Resultados: En todos los casos en los que se encontraron factores de riesgo (género femenino, hipertensión, diabetes, dislipidemia y tabaquismo), el índice de SLG fue subnormal, al igual que en todos los casos con remodelación concéntrica, hipertrofia concéntrica y excéntrica; también en todos aquellos casos con disfunción diastólica caracterizados por relajación prolongada o patrón pseudonormal así como con presencia de dilatación de aurícula izquierda. Anotándose que en todos los casos la fracción de eyección del ventrículo izquierdo se encontró en parámetros normales. Conclusiones: La reducción de SLG se correlaciona con el grado de disfunción diastólica, las anormalidades en las mediciones convencionales ecocardiográficas, al igual que con la presencia de riesgos cardiovasculares. Se le considera un parámetro predictor adverso.

Palabras clave: Deformidad longitudinal global, factores de riesgo cardiovascular, mediciones electrocardiográficas.

\section{Abstract}

Introduction: Prognosis in patients with cardiovascular structural disease largely depends on the left ventricular function quality; the measurement of myocardial deformation (strain) is an earlier and more sensitive indicator of heart failure. Objective: To correlate the left ventricle diastolic dysfunction degree and other 2D echocardiogram variables with the global longitudinal strain (SLG). Material and methods: 53 patients with an average age and SD of 59.8 years \pm 17.5 and a range of 22 to 91 years were studied, in which routine echocardiographic and SLG measurements were made. Statistical analysis: $\chi^{2}, \mathrm{t}$ Student, and odds ratio were used to assess the relationship of the variables with the SLG. Results: In all cases where diastolic dysfunction was found characterized by prolonged relaxation or pseudo normal filling pattern the SLG was subnormal; likewise, in all cases with concentric remodeling, concentric and eccentric hypertrophy, as well as with the presence of left atrial dilation; in the same way in all cases where risk factors were found (female gender, systemic hypertension, diabetes, dyslipidemia, and smoking) the SLG index was subnormal. Conclusions: The reduction of global longitudinal strain correlates with the degree of diastolic dysfunction, abnormalities in conventional echocardiographic measurements, as well as the presence of cardiovascular risk factors, all of which should be considered as adverse prognostic predictors.

Keywords: Global longitudinal strain, cardiovascular risk factors, echocardiographic measurements.

\footnotetext{
* Cardiólogo, Jefe de Ecocardiografía del Hospital Angeles León. León, Guanajuato, México.

₹ Especialista en Medicina de Rehabilitación, Catedrático de la Facultad de Medicina de León, Universidad de Guanajuato. México.

§ Médico pasante del Servicio Social, Universidad Anáhuac México. México.

" Médico pasante del Servicio Social, Universidad Autónoma de Guadalajara. México.
}

Correspondencia:

Dr. Francisco Sánchez Lezama

Correo electrónico: sanchezlezamafco72@gmail.com

Aceptado: 15-01-2021.

www.medigraphic.com/actamedica

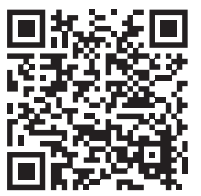




\section{INTRODUCCIÓN}

La enfermedad cardiovascular es la principal causa de muerte en todo el mundo. Se estima que casi $30 \%$ de todas las muertes en el mundo son causadas por esta enfermedad. ${ }^{1}$ Hace algunos años se asumía que la insuficiencia cardiaca era consecuencia de la pérdida de la capacidad contráctil del corazón, centrándose en la función sistólica. últimamente se ha observado que los pacientes con signos y síntomas de insuficiencia cardiaca (alrededor de 50\%) tienen función sistólica ventricular conservada, lo que nos lleva a dar más importancia a la función diastólica del ventrículo izquierdo. ${ }^{2,3}$ Se utiliza el término de insuficiencia cardiaca diastólica cuando aparecen signos o síntomas en un paciente con disfunción diastólica (DD); los principales factores de riesgo del DD son: género femenino, edad mayor de 65 años, hipertensión arterial, diabetes mellitus e hipertrofia ventricular izquierda. ${ }^{4}$ En estos pacientes se incrementa la morbilidad y mortalidad. La ecocardiografía Doppler ha sido ampliamente utilizada de manera eficaz para la evaluación de la función diastólica; ${ }^{5}$ sin embargo, los parámetros ecocardiográficos convencionales para su evaluación tienen menor sensibilidad para la evaluación exhaustiva de la contractilidad miocárdica, ya que no detectan pequeños cambios en la contractilidad o alteraciones. ${ }^{6}$ Con el avance de la técnica de ecocardiografía speckle-tracking bidimensional (2D-STE) utilizada para el análisis de la deformación miocárdica, mediante el seguimiento automático del movimiento de patrones de puntos inherentes a la interfase ultrasonido-miocardio ${ }^{7}$ es posible evaluar la contractilidad del ventrículo izquierdo, ya que captura y rastrea puntos del ecocardiograma bidimensional a lo largo del ciclo cardiaco, generando vectores de movimiento y curvas de deformación (strain y strain rate) en los planos ortogonales longitudinal, radial y circunferencial, obteniendo un índice clínico de deformación miocárdica relacionado con la longitud al final de la diástole, lo que permite cuantificar tanto la magnitud como el tiempo de deformación regional, sistólica y diastólica.

Dada la evidencia de que la disfunción diastólica está asociada con fibrosis miocárdica, es posible considerar que esta disfunción puede indicar daño prematuro del músculo cardiaco, reconociendo que la deformación longitudinal global (SLG) permite identificar etapas incipientes de daño miocárdico, se puede asumir que la diastólica es un marcador temprano de miocardiopatía, y su evolución sin intervención médica conducirá a un estado de cardiopatía avanzada. ${ }^{8}$

El objetivo de este estudio fue correlacionar el grado de disfunción diastólica y otras variables del ecocardiograma 2D con el índice de deformación longitudinal global (SLG por sus siglas en inglés strain longitudinal global) mediante speckle tracking.

\section{MATERIAL Y MÉTODOS}

La muestra estuvo conformada por 53 pacientes consecutivos, 32 mujeres y 21 hombres. Se incluyeron todos los pacientes con fracción de eyección del VI normal $\geq$ $50 \%$ y ritmo sinusal, excluyendo aquéllos con cardiopatía isquémica y/o miocardiopatía. Todos los estudios fueron realizados por un cardiólogo certificado en ecocardiografía. Las mediciones convencionales en ecocardiografía en modo M, Doppler tisular en color bidimensional, pulsado, continuo y los informes de deformación longitudinal global (SLG) se realizaron utilizando un ecocardiógrafo Philips modelo EPIQ 7C o Philips CX50, con transductor multifrecuencia de 1-3 MHz, con especial énfasis en la estructura y tamaño de la aurícula izquierda. Se registraron los resultados y las mediciones de los perfiles demográficos y de factores de riesgo cardiovascular. Las variables obtenidas fueron: edad, sexo, tabaquismo, antecedentes de hipertensión arterial sistémica, diabetes mellitus, dislipidemia; diámetro diastólico del ventrículo izquierdo (DDVI), diámetro sistólico del ventrículo izquierdo (DSVI), espesor final (EF) y fracción de eyección del VI (FEVI). Las medidas específicas fueron: relación espesor/radio de la telediástole del $\mathrm{VI}(\mathrm{T} / \mathrm{R})$, que se obtuvo mediante la medición del eco en modo M: espesor de la pared posterior $\mathrm{x}$ 2/diámetro diastólico; el valor normal se estableció en < 0.42 . Masa del ventrículo izquierdo, calculada en modo M utilizando la siguiente ecuación: ${ }^{9} 1.04 \times(0.8 \times([\mathrm{DDV})$ + IVST + PWT ] 3 - [DDVI] 3) + 0.6; se consideró valor normal $<95 \mathrm{~g} / \mathrm{m}^{2}$ ASC en mujeres y $<115 \mathrm{~g} / \mathrm{m}^{2}$ ASC en hombres. De acuerdo con esto, se consideraron cuatro geometrías ventriculares diferentes: 1 ) normal (masa normal y relación $\mathrm{T} / \mathrm{R}$ normal); 2) remodelado concéntrico (masa normal y aumento de la relación T/R); 3) hipertrofia concéntrica (masa aumentada y relación $\mathrm{T} / \mathrm{R}$ aumentada) y 4) hipertrofia excéntrica (masa aumentada y relación $T / R$ normal).

El volumen de la aurícula izquierda (Al) se obtuvo mediante el método biplano de Simpson en vistas apicales de dos y cuatro cámaras, utilizando el software integrado de la máquina. Para este propósito, las áreas de la Al se trazaron manualmente al final de la sístole en vistas apicales de cuatro y dos cámaras, asegurando que no hubo acortamiento de la Al. El cálculo automático del volumen se realizó utilizando el software para el método de suma de disco de Simpson modificado en la máquina de eco. El volumen de la $\mathrm{Al}$ se indexó al área de superficie corporal y se expresó como índice de volumen de $\mathrm{Al}\left(\mathrm{mL} / \mathrm{m}^{2}\right.$ BSA); el valor normal del volumen de la aurícula izquierda fue $<29 \mathrm{~mL} / \mathrm{m}^{2}$ BSA. En la vista apical de cuatro cámaras, utilizando un volumen de muestra de 1-2 mm, el cursor Doppler de 
onda de pulso (PW) se coloca entre las puntas de las valvas mitrales durante la diástole. A partir del perfil de flujo de entrada mitral se midió la onda E. El promedio de las velocidades diastólica lateral y septal temprana (e') se midió mediante imágenes de Doppler tisular de la vista apical de cuatro cámaras. Se calculó la relación E/e' y se consideró un valor $\geq 15$ como indicativo de presiones de llenado del VI elevadas. ${ }^{5}$

Un solo investigador realizó el análisis bidimensional de speckle tracking en las tres proyecciones apicales, primero, rastreando manualmente el endocardio al inicio de la sístole, después de lo cual el software siguió el patrón de motas del miocardio cuadro por cuadro. La región de interés se ajustó para cubrir el grosor del miocardio, se verificó y corrigió el seguimiento adecuado si era necesario. El análisis de deformación se realizó sólo si el software podía rastrear más de cuatro de seis segmentos. Posteriormente, el software dividió el VI en 18 segmentos. Se calculó la deformación longitudinal global (SLG) para cada una de las tres proyecciones apicales y para la SLG media como el promedio de las tres proyecciones. Para los fines de este estudio, el valor de SLG se informó en signo negativo para facilitar la interpretación y el punto de corte, determinándolo en $-18.0 \%$.

Análisis estadístico. Se utilizó $\chi^{2}$ con corrección de Yates, ya que la muestra fue menor de 200 individuos para relacionar variables cualitativas así como t de Student para relacionar variables cuantitativas-cualitativas; se consideró significativo el valor de $p=0.05$; se obtuvo la OR (odds ratio) para evaluar la relación entre factor de riesgo y SLG.

\section{RESULTADOS}

La muestra estudiada estuvo constituida por 53 pacientes (Tabla 1), de los cuales 32 correspondieron al género femenino (60.3\%) con una edad media y DE de $61.7 \pm 17$ y un rango de 22 a 91 años; al género masculino 21 (39.7\%) con edad media y DE de $57.8 \pm 18.55$ y rango de 24 a 84 años; la relación entre géneros fue de 1.5:1 a favor del femenino, sin diferencia estadísticamente significativa entre géneros, pero con presencia de cinco años promedio mayor en el femenino.

Las mediciones ecocardiográficas y su correlación con el SLG en 53 pacientes se reportan en la Tabla 2. En cuanto a la relación entre SLG y función diastólica del VI, se observó que cuando era normal $(n=24), 45.3 \%$, el SLG presentó un promedio de $-18.55 \%(p=0.005)$; en presencia de relajación prolongada $(n=20)(37.8 \%)$, el SLG disminuyó a $-17.16 \%$ con OR de 1.1 ( $p=0.05$ ); en los casos clasificados como pseudonormales $(n=9)(16.9 \%)$, el valor de SLG fue incluso menor $-15.52 \%$, con OR de $0.9(p=0.05)$.

Con relación al patrón de geometría ventricular normal $(\mathrm{n}=14)(26.4 \%)$, el SLG correspondió en promedio a -18.55\%; cuando se observó remodelado concéntrico ( $\mathrm{n}=$ 27) (50.94\%), el SLG correspondió a $-17.27 \%$ en promedio con OR de $1.6(p=0.05)$; en presencia de hipertrofia concéntrica ( $n=11,20.75 \%$ ), el valor de SLG fue $-16.03 \%$ con un OR de 0.9 ( $p=0.04)$; sólo se encontró un caso con hipertrofia excéntrica y el SLG correspondiente a $-15.20 \%$.

Las mediciones del volumen de la aurícula izquierda y del volumen indexado mostraron dilatación de la aurícula

\begin{tabular}{lccccc}
\multicolumn{2}{c}{ Tabla 1: Datos demográficos, factores de riesgo cardiovascular y su correlación } \\
con medición de deformidad miocárdica global en 53 pacientes.
\end{tabular}


Tabla 2: Mediciones ecocardiográficas y su correlación con la deformidad longitudinal global en 53 pacientes.

\begin{tabular}{|c|c|c|c|c|c|c|}
\hline Variable & $\mathrm{n}(\%)$ & Media $\pm \mathrm{DE}$ & Rango & SLG, \% & OR & $p$ \\
\hline GSIV (mm) & $53(100.00)$ & $11.19 \pm 1.82$ & $8.7-15.4$ & NR & - & - \\
\hline GPP (mm) & $53(100.00)$ & $10.17 \pm 0.42$ & $8.0-11.9$ & NR & - & - \\
\hline DDVI (mm) & $53(100.00)$ & $41.79 \pm 7.14$ & $22-56$ & NR & - & - \\
\hline DSVI (mm) & $53(100.00)$ & $22.86 \pm 6.19$ & $8.3-36$ & NR & - & - \\
\hline \multicolumn{7}{|l|}{ Geometría ventricular } \\
\hline Normal & $14(26.40)$ & - & - & -18.76 & - & - \\
\hline $\mathrm{RC}$ & $27(50.94)$ & - & - & -17.27 & 1.4 & 0.050 \\
\hline $\mathrm{HC}$ & $11(20.75)$ & - & - & -16.03 & 1.1 & 0.050 \\
\hline $\mathrm{HE}$ & $1(1.89)$ & - & - & -15.20 & - & - \\
\hline \multicolumn{7}{|l|}{ Flujo transmitral } \\
\hline $\mathrm{E}(\mathrm{cm} / \mathrm{s})$ & $53(100.00)$ & $84.62 \pm 24.74$ & $50-150$ & - & - & - \\
\hline $\mathrm{e}^{\prime}(\mathrm{cm} / \mathrm{s})$ & $53(100.00)$ & $8.69 \pm 2.81$ & $5.5-17.2$ & - & - & - \\
\hline Ele' & $53(100.00)$ & $10.64 \pm 4.41$ & $5.1-18$ & - & - & - \\
\hline \multicolumn{7}{|l|}{ Función diastólica } \\
\hline Normal & $24(45.30)$ & - & - & -18.55 & - & 0.050 \\
\hline PRP & $20(37.80)$ & - & - & -17.16 & 1.2 & 0.050 \\
\hline PPN & $9(16.90)$ & - & - & -15.52 & 0.9 & 0.050 \\
\hline $\mathrm{VAI}(\mathrm{mL})$ & $53(100.00)$ & $54.98 \pm 20.01$ & $24-105$ & - & - & - \\
\hline Al dilatada & $11(20.70)$ & - & - & -15.22 & 1.1 & 0.003 \\
\hline Al indexada $\left(\mathrm{mL} / \mathrm{m}^{2} \mathrm{SC}\right)$ & $53(100.00)$ & $31.17 \pm 12.32$ & $17.1-61.0$ & - & - & 0.003 \\
\hline SLG/muestra & $53(100.00)$ & $-17.51 \%$ & $12.2-22.4$ & - & - & - \\
\hline SLG/femenino & $32(60.37)$ & $-12.50 \pm 13.39$ & $-13.0-122.4$ & - & - & 0.050 \\
\hline SLG/masculino & $21(39.63)$ & $-16.71 \pm 2.36$ & $-12.2--20.8$ & - & - & 0.050 \\
\hline SLG/muestra (> -18\%) & $25(47.16)$ & -13.05 & $-12.2--17.9$ & - & - & 0.004 \\
\hline SLG/femenino (>-18\%) & $12(37.50)$ & -10.64 & $-13-0--17.9$ & - & 0.8 & - \\
\hline SLG/masculino (> -18\%) & $13(61.90)$ & -15.27 & $-12.2--17.3$ & - & 0.9 & - \\
\hline FEVI & $53(100.00)$ & $71.71 \pm 4.49$ & $59-80$ & - & - & - \\
\hline
\end{tabular}

$\mathrm{DE}=$ desviación estándar; SLG = strain longitudinal global; OR = odds-ratio; GSIV = grosor del septum interventricular; GPP = grosor de pared posterior; $\mathrm{DDVI}$ = diámetro diastólico del ventrículo izquierdo; $\mathrm{DSVI}=$ diámetro sistólico del ventrículo izquierdo; $\mathrm{RC}$ = remodelación concéntrica; $\mathrm{HC}=$ hipertrofia concéntrica; $\mathrm{HE}$ = hipertrofia excéntrica; $\mathrm{PRP}=$ patrón de relajación prolongada; $\mathrm{PPN}$ = patrón pseudonormal; VAl = volumen de aurícula izquierda; $\mathrm{Al}$ = aurícula izquierda; $\mathrm{FEVI}$ = fracción de eyección del ventrículo izquierdo.

izquierda en 11 pacientes (20.75\%), que se relacionó con un SLG de $-15.22 \%$.

En cuanto a los factores de riesgo presentes correlacionados con una disminución del índice de deformidad longitudinal global como hipertensión arterial, diabetes mellitus, dislipidemia y tabaquismo, se observó que la hipertensión arterial en $62.2 \%$ de la muestra $(n=33), 23$ casos $(69.7 \%)$ correspondieron al género femenino y 10 (30.3\%) al masculine. En este grupo de pacientes la SLG promedio se observó en $-17.32 \%$ con una razón de desventaja OR (odds ratio) de $1.6(p=0.005)$. En relación con la presencia de diabetes mellitus, la SLG promedio fue de $-16.41 \%$ con una OR de $0.8(p=0.05)$, predominando el género femenino con una relación de 9:1. La presencia de dislipidemia se encontró en 13 casos y la SLG promedio fue de $-17.50 \%$ con OR de $0.9(p=0.05)$, sin diferencia estadística entre géneros; se encontró tabaquismo en 10 casos (18.9\%) y en este grupo la SLG fue de $-16.58 \%$ con OR de 0.8 ( $p=0.05)$.

En 100\% del grupo estudiado la fracción de eyección del ventrículo izquierdo fue normal con un valor promedio y DE de $71.71 \% \pm 4.49 \%$ y una variación de 59 a $80 \%$.

\section{DISCUSIÓN}

Habitualmente la determinación de la fracción de eyección del ventrículo izquierdo (FEVI) es el método utilizado para medir la función sistólica; sin embargo, con la llegada del siglo XXI y el avance de la tecnología ecocardiográfica se logró la medición de la deformación (strain) miocárdica en sus diferentes variedades desde el año 2010,10,11 así como la mejora del equipamiento a lo largo del tiempo y la estandarización de las mediciones a realizar. ${ }^{12}$ El abordaje 
diagnóstico ecocardiográfico se ha incrementado sobre todo en la última década, mejorando las mediciones del miocardio tanto de su estructura como de su valoración functional. ${ }^{13}$ Actualmente se considera imprescindible el análisis de la deformación miocárdica como parte del estudio ecocardiográfico del corazón. ${ }^{14}$

En cuanto al grupo estudiado en este informe, los cambios estructurales y funcionales detectados por la medición del índice de deformidad longitudinal global se correlacionan directamente con la presencia de factores de riesgo cardiovascular, ya que el índice SLG reportó cifras por debajo del punto de corte $(I=-18.0 \%)$ en la mayoría de los pacientes mayores de 65 años, con predominio del género femenino, destacando en este trabajo que la relación de género fue de 1.5: 1 a favor del primero, dato que concuerda con la literatura. ${ }^{15}$ En cuanto a la presencia de hipertensión arterial, también fue más frecuente en el género femenino con una relación 2.3:1, encontrando en todos los casos el índice SLG por debajo del punto de corte $(\mathrm{p}=0.005)$, también mostró odds ratio (OR) de 1.6, siendo consistente con el reporte de Villarroel y colaboradores. ${ }^{16}$ En cuanto a diabetes mellitus (DM), en este reporte llama la atención que el género femenino predominó con una relación 9:1. Se observó que en este grupo el índice SLG fue significativamente menor que en pacientes con otros factores de riesgo, por lo que el SLG es un parámetro temprano de cardiopatía en presencia de DM, siendo estadísticamente significativo ( $p=0.05)$ y OR de 1.1; datos que son corroborados por varios estudios a lo largo del tiempo. ${ }^{17-19}$ En cuanto a los pacientes con dislipidemia, se observó, como en las variables anteriores, un predominio del género femenino con relación 1.5:1, detectando una discreta, pero presente disminución en el índice SLG sin significancia estadística $(p=0.06)$, posiblemente por el tamaño pequeño de la muestra. Por el contrario, cuando coexisten DM e hyperlipidemia, hubo mayor disminución de SLG como lo reportan Vinereanu y colaboradores, ${ }^{20}$ la disminución del SLG ya está presente como dato subclínico en este grupo de pacientes. Con relación al tabaquismo, fue la única variable predominante en el género masculino, afectando la disminución de SLG en todos los casos con significancia estadística $p=0.05$ y OR de 0.8 .

Respecto a las medidas ecocardiográficas cabe destacar: 1) el patrón de geometría ventricular que presentaban los pacientes, tanto de remodelación concéntrica $(\mathrm{OR}=1.4)$ como de hipertrofia concéntrica $(\mathrm{OR}=1.1)$, mostró valores por debajo del punto de corte (ambos con $p=0.05$ ), con mayor disminución en el último, este dato concuerda con el reporte de Saito y su equipo, ${ }^{21}$ en un solo caso de hipertrofia excéntrica se observó el valor más bajo de la muestra; 2) la función diastólica anormal, como la relajación prolongada $(\mathrm{OR}=1.2)$ y el patrón pseudonormal (OR
$=0.9$ ), se relacionó directamente con valores inferiores al punto de corte del SLG, obteniendo valores más bajos con el patrón pseudonormal; ambos resultaron estadísticamente significativos $(p=0.05) ; 3)$ la dilatación de la aurícula izquierda tanto en su volumen absoluto medido $(\mathrm{OR}=1.1)$ como cuando se indexó a la superficie corporal $(\mathrm{OR}=1.2)$, mostró que en 11 casos el SLG presentó cifras por debajo del punto de corte $(p=0.003)$, lo que concuerda con lo indicado en el estudio de Sherazi y Zareba; ${ }^{3}$ por tanto, se puede inferir que el índice SLG asociado al hallazgo de dilatación auricular izquierda ${ }^{5}$ resultan ser los parámetros ecocardiográficos precoces en la disfunción cardiaca.

Cabe destacar que en este trabajo no se encontró relación entre el SLG y los diámetros diastólico y sistólico del VI. Por otro lado, la fracción de eyección del VI en todos los casos estudiados estuvo dentro de los valores normales. Esto último se explica porque la mecánica longitudinal del ventrículo izquierdo depende predominantemente del subendocardio, que es el más vulnerable y sensible a la presencia de enfermedad miocárdica. La función de las capas media y epicárdica puede permanecer relativamente normal en un inicio, por lo que la deformación circunferencial y la torsión pueden permanecer iguales o incluso tener un comportamiento compensatorio exagerado para mantener la función sistólica, mientras que la disminución de la distensibilidad ventricular produce alteración de la relajación longitudinal ventricular y retraso de distorsión progresiva, que altera la función diastólica al elevar las presiones de llenado en una fase en la que la fracción de eyección aún es normal. ${ }^{22-24}$

Por último, el desarrollo e incorporación de la medición ecocardiográfica del índice de deformidad longitudinal ha demostrado una enorme utilidad en diversas patologías. Día a día esta técnica se suma a situaciones como el infarto agudo de miocardio sin elevación del $\mathrm{ST}^{25}$ y a la valoración de las repercusiones cardiacas con el uso de quimioterapia, ${ }^{26}$ permitiendo la detección precoz de alteraciones así como su seguimiento y pronóstico, por lo que representa un amplio campo de investigación.

La desventaja de este reporte es que la muestra es pequeña; sin embargo, los datos estadísticos calculados indican relaciones directas y concretas, las cuales son consistentes con reportes de otras latitudes. No obstante, se requiere mayor número de pacientes para confirmar los hallazgos de este estudio.

\section{CONCLUSIÓN}

La alteración de la deformación miocárdica (strain) es un indicador temprano y sensible de insuficiencia cardiaca y se correlaciona con el grado de disfunción diastólica y otras variables estudiadas en el ecocardiograma 2D conven- 
cional, así como con los factores de riesgo cardiovascular tradicionales. Su cuantificación implica el diagnóstico, seguimiento, toma de decisiones terapéuticas y pronóstico de diversas patologías cardiacas.

\section{REFERENCIAS}

1. GBD 2013 Mortality and Causes of Death Collaborators. Global, regional, and national age-sex specific all-cause and cause-specific mortality for 240 causes of death, 1990-2013: a systematic analysis for the Global Burden of Disease Study 2013. Lancet. 2015; 385(9963): 117-171.

2. Park JJ, Park JB, Park JH, Cho GY. Global longitudinal strain to predict mortality in patients with acute heart failure. J Am Coll Cardiol. 2018; 71(18): 1947-1957. doi: 10.1016/j.jacc.2018.02.064.

3. Sherazi S, Zareba W. Diastolic heart failure: predictors of mortality. Cardiol J. 2011; 18(3): 222-232.

4. Díaz Herrera W, Rincón Flórez DF, Martínez Montalvo CM. Evaluación de la disfunción diastólica y consideraciones perioperatorias. Acta Méd Peru. 2017; 34(3): 208-216.

5. Sánchez-Lezama F, Domínguez-Carrillo LG, Harrison-Gómez C, Ramírez-Lagundas M. Clinical and echocardiographic variables associated to left atrium enlargement. Differences between genders. Cardiovasc Metab Sci. 2019; 30(1): 6-13.

6. Del Castillo JM, Herszkowicz N, Ferreira C. Speckle tracking. A contratilidade miocárdica em sintonia fina. Rev Bras Ecocardiogr Imagem Cardiovasc. 2010; 23(3): 46-54.

7. Mondillo S, Galderisi M, Mele D, Cameli M, Lomoriello VS, Zaca V et al. Speckle-tracking echocardiography: a new technique for assessing myocardial function. J Ultrasound Med. 2011; 30(1): 71-83.

8. Cuevas RJ, Lacambra BI. Ecocardiografía avanzada: aplicación clínica actual del análisis de deformación miocárdica y de la imagen tridimensional. Rev Ecuat Med Cienc Biol. 2017; 38(2): 67-83. Disponible en: doi.org/10.26807/remcb.v38i2.550

9. Devereux RB, Alonso DR, Lutas EM, Gottlieb GJ, Campo E, Sachs I et al. Echocardiographic assessment of left ventricular hypertrophy: comparison to necropsy findings. Am J Cardiol. 1986; 57(6): 450-458.

10. D'hooge J, Heimdal A, Jamal F, Kukulski T, Bijnens B, Rademakers $\mathrm{F}$ et al. Regional strain and strain rate measurements by cardiac ultrasound: principles, implementation and limitations. Eur J Echocardiogr. 2000; 1(3): 154-170.

11. Urheim S, Edvardsen T, Torp H, Angelsen B, Smiseth OA. Myocardial strain by Doppler echocardiography. Validation of a new method to quantify regional myocardial function. Circulation. 2000; 102(10): 1158-1164.

12. Yingchoncharoen T, Agarwal S, Popović ZB, Marwick TH. Normal ranges of left ventricular strain: a meta-analysis. J Am Soc Echocardiogr. 2013; 26(2): 185-191.

13. Rodríguez $\mathrm{BI}$, Jiménez NM, Pérez GR, García OR, Morillo VE, de Teresa GE. Deformación ventricular izquierda en ecocardiografía bidimensional: valores y tiempos en sujetos normales. Rev Esp Cardiol. 2010; 63(10): 1195-1199. doi: 10.1016/S0300-8932(10)70252-9.
14. Voigt JU, Pedrizzetti G, Lysyansky P, Marwick TH, Houle H, Baumann $R$ et al. Definitions for a common standard for 2D speckle tracking echocardiography: consensus document of the EACVI/ASE/Industry Task Force to standardize deformation imaging. Eur Heart J CardiovasC Imaging. 2015; 16(1): 1-11.

15. Cheng S, Larson MG, McCabe EL, Osypiuk E, Lehman BT, Stanchev $P$ et al. Age- and sex-based reference limits and clinical correlates of myocardial strain and synchrony: the Framingham Heart Study. Circ Cardiovasc Imaging. 2013; 6(5): 692-699.

16. Villarroel-Ábrego H, Garillo R, González-Suero JC, Núñez AE. Correlación entre fracción de eyección del ventrículo izquierdo y strain longitudinal global en pacientes con hipertensión arterial. Insuf Card. 2018; 13(2): 57-66.

17. Ng AC, Delgado V, Bertini M, van der Meer RW, Rijzewijk LJ, Shanks $M$ et al. Findings from left ventricular strain and strain rate imaging in asymptomatic patients with type 2 diabetes mellitus. Am J Cardiol. 2009; 104(10): 1398-1401.

18. Faden G, Faganello G, De Feo S, Berlinghieri N, Tarantini L, Di Lenarda A et al. The increasing detection of asymptomatic left ventricular dysfunction in patients with type 2 diabetes mellitus without overt cardiac disease: data from the SHORTWAVE study. Diabetes Res Clin Pract. 2013; 101(3): 309-316.

19. Holland DJ, Marwick TH, Haluska BA, Leano R, Hordern MD, Hare JL et al. Subclinical LV dysfunction and 10-year outcomes in type 2 diabetes mellitus. Heart. 2015; 101(13): 1061-1066.

20. Vinereanu D, Nicolaides E, Tweddel AC, Madler CF, Holst B, Boden LE et al. Subclinical left ventricular dysfunction in asymptomatic patients with Type II diabetes mellitus, related to serum lipids and glycated haemoglobin. Clin Sci (Lond). 2003; 105(5): 591-599.

21. Saito M, Okayama H, Yoshii T, Higashi H, Morioka H, Hiasa G et al. Clinical significance of global two-dimensional strain as a surrogate parameter of myocardial fibrosis and cardiac events in patients with hypertrophic cardiomyopathy. Eur Heart J Cardiovasc Imaging. 2012; 13(7): 617-623.

22. Pinto M. Strain: una ventana a la mecánica ventricular. Rev Chil Cardiol. 2011; 30(2): 155-159.

23. Cañón-Montañez W, Santos A, Foppa M. Strain longitudinal global: un parámetro útil para evaluar disfunción ventricular izquierda subclínica en el síndrome metabólico. Rev Colomb Cardiol. 2016; 23(2): 112-119. Disponible en: http://dx.doi.org/10.1016/j. rccar.2015.10.008

24. Herrera-Márquez R, Peralta-Cortázar C, Contreras-Rodríguez A, Hernández-Rodríguez J, Manjarrez-Gutiérrez G. Disfunción diastólica subclínica del ventrículo izquierdo en adolescentes con diabetes tipo 1. Bol Med Hosp Infant Mex. 2014; 71(3): 142-147.

25. Salazar-Marín S, Valencia JM, Hernández-Vásquez OM, Estrada JM. Utilidad del strain sistólico pico longitudinal bidimensional en pacientes con diagnóstico clínico de infarto del miocardio sin elevación del ST. Rev Colomb Cardiol. 2017; 24(6): 550-558.

26. Reborido N, Parma G, Noria S, Schiavone A, Bonelli A, Lluberas R. Strain bidimensional para detección precoz de disfunción sistólica del ventrículo izquierdo inducida por agentes quimioterápicos. Rev Urug Cardiol. 2016; 31(2): 266-276. 